\title{
Characteristics of Prisoners with Intellectual Disabilities
}

\begin{abstract}
\section{Background}

Previous studies have found high rates of intellectual disabilities (ID) in prison. However, little is understood about prisoners with ID. This study aimed to identify prisoners with ID and compare their characteristics with prisoners without neurodevelopmental disorders with regards to demographic profile, mental health, suicide risk and offences.
\end{abstract}

\section{Method}

This was a descriptive, cross-sectional study carried out using face-to-face interviews with 240 participants in a London Category C prison. Standardised tools were used to assess prisoners for ID and mental disorder.

\section{Results}

The study identified 18 prisoners as having ID. Participants with ID were less likely to be from a black or minority ethnic background, be over 35 years of age or have any qualifications. They were more likely to have been single, homeless or unemployed before coming into prison. Prisoners with ID were significantly more likely to have mental health problems and $25 \%$ had thought about suicide in the last month and 63\% had attempted suicide in the past. Prisoners with ID were also more likely to be housed in the vulnerable prisoners wing and significantly more likely to have committed robbery than other prisoners.

\section{Conclusions}

The findings confirm the presence of significant numbers of people with ID with high levels of mental illness in a male prison. Services across the CJS are required for this group, specifically, there is a need for: raised awareness among those working in prison about ID, and improved skills to recognise offenders with ID and address major gaps in current healthcare provision in prison.

Keywords: intellectual disabilities, forensic mental health, mental illness, prison, neurodevelopmental disorders, suicide 


\section{Introduction}

The prevalence of intellectual disability (ID) in prisoners is estimated to be $11 \%$ of remand and 5-7\% of sentenced prisoners (Singleton et al, 1998; Talbot, 2007; Talbot, 2008; Murphy etal., 2015). This could equate to over 6000 prisoners in England and Wales (Ministry of Justice, 2014) and compares to 1-3\% of people in the general population with ID; (Harris, 2006). Although this study is UK based it has implications for the penal system across countries.

Data from the Psychiatric morbidity in prisoners with intellectual disabilities: analysis of prison survey data for England and Wales, found higher rates of psychotic disorder and common mental health problems, such as cannabis abuse, suicidality and self-harm compared with the rest of the prison population (Hassiotis et al, 2011). However, the evidence base on the mental health of prisoners with ID is still limited. In the UK there has been a plethora of policy and guidance relating to people with ID at risk of contact with or in the Criminal Justice System including the Bradley Report (Ministry of Justice, 2009) and Building the Right Support (NHS England, 2015) as part of the Transforming Care agenda. However there is little evidence to suggest there has been any sustained success in diverting people with ID from prison in spite of policy and increased awareness of the problem through reports highlighting the injustices faced by prisoners with ID such as No One Knows (Talbot, 2008). Despite a general agreement and recommendations for the need to provide screening to detect people with ID prison, there is little evidence of commisioned services for this group of people. The principle of equivalence of care, i.e prisoners are entitled to the same standard of healthcare as to those outside prison is not a reality in prison healthcare for people with ID and those with mental health problems and adds further weight for the need to divert people with ID from to meet the needs of this vulnerable group.

This paper focuses on trying to increase understanding of prisoners with ID and examines characteristics that increase vulnerability in this group i.e. suicide risk, comorbid mental disorder and neurodevelopmental disorder (ND) by comparing ID prisoners to prisoners without ID or other NDs. Central to this study is the use of clinical interviews, with secondary data examined to verify prisoner's experiences and offence profiles.

In general people with ID are a more vulnerable to bullying and are less able to cope within the prison environment (Talbot, 2008) and it is likely that those with co-occurring health problems are at increased risk of further offending behaviour (Chaplin et al, 2013). The presence of ID has implications for release and parole; in particular, for those required to engage in treatment that they do not understand because of reduced cognitive abilities (Straw \& Lomri, 2010). The Bradley review (Ministry of Justice, 2009) of people with mental health problems or ID in the 
United Kingdom Criminal Justice System focussed on early detection, intervention and diversion from prison and acknowledged that offender treatment programmes within prisons are often not accessible to people with ID. Prison health pathways will differ by establishment and levels of security often with no specific support for people identified with ID outside of mental health in reach team.

\section{Method}

The study was approved by the National Research Ethics Service Committee North East Northern \& Yorkshire (ref: 12/NE/0040), the National Offender Management Service (NOMS; ref: 50-12).

\section{Setting}

The study was carried out in a category C prison, in south London. At the time of study, the prison was run by National Offender Management Service and had a maximum capacity of 798 prisoners. When the data for the study were collected, mental health care in the prison was provided by general practitioners, a primary care mental health team, an 'outreach' team (psychiatrists, community psychiatric nurses and a psychologist), an Improving Access to Psychological Therapies Service (IAPTS), counsellors and a substance misuse team.

\section{Participants}

Eligibility criteria for the study were that participants were detained in the prison, demonstrated capacity to consent to take part and were over 18 years of age. For the purposes of this study, participants with ID were defined as those who scored less than $46 \%$ on the Learning Disability Screening Questionnaire (LDSQ) during a screening interview. Mental health data on prisoners screening positive for ID was sought for comparison with a control group. This 'control' group were selected according to whether they could be located for follow-up interview and had agreed to provide further information about their mental health.

In total, $378(18 \%)$ of prisoners who arrived during the study period between May 2012 and June 2013. Of those, 240 prisoners (63\%) consented to take part of these $15(6 \%)$ self-referred to the study and a further 39 participants (16\%) were referred by prison healthcare or education staff. (Figure 1 illustrates the flow of participants through the study). The participants screened during the course of the project were representative of the broader population of the study prison in terms of age and ethnicity, i.e., around half were from Black and Minority Ethnic (BME) backgrounds and just over two-thirds were under 40 years of age replicating findings from Her Majesty's Chief Inspector of Prisons, 2013). Of those who took part, 211 (88\%) were 
sentenced prisoners and $29(12 \%)$ were on remand.

\section{Procedure}

An assessment of capacity was conducted to ensure potential participants were eligible for the study.

\section{Measures}

The Learning Disability Screening Questionnaire (LDSQ) (McKenzie et al, 2012), self report and case note review was used to screen for ID. The LDSQ has a reported sensitivity and specificity of over $80 \%$. The LDSQ comprises seven items, $<46 \%$ indicates the presence of ID. A range of sociodemographic data were collected during screening interviews as well as information on any existing or previous diagnoses of ND, mental health problems or substance abuse. As part of the wider study, in addition to ID screening, the study also measured symptoms of Attention Deficit Hyperactivity Disorder (ADHD) and Autism Spectrum Disorder (ASD) using the ADHD adult rating scale (ASRS) (Adler et al, 2008) and 20-item autism quotient (AQ-20) (Brugha et al, 2012). Participants who screened positive for ID on the LDSQ were assessed for suicidality, mental health problems and substance misuse using the Mini International Neuropsychiatric Interview (MINI v.6.0.0; Sheehan et al, 2010). A 'control' group of 69 participants without ID, ADHD or ASD (i.e. participants who screened negative on the LDSQ, ASRS and AQ-20) were also assessed using the MINI. This group are referred to having no ND. Interview and case note review of information held on P-NOMIS and System one (the prison records systems) was carried out to gain the offence history of participants.

\section{Analysis}

Chi-square tests were used to analyse the data, using SPSS version 20.

\section{Results}

Of the 240 prisoners who took part in the study18 (7.5\%) were identified by the LDSQ as likely to have ID (see figure 1). Of those who screened positive on the LDSQ, 16 (89\%) also selfreported having ID or learning difficulties. Of these $11(60 \%)$ had previously received ID services. Prior to the study only $4(22 \%)$ had been identified as having an ID in the prison records. The nature of learning difficulties was not always clear and may have met the threshold for ID given how it was described by the prisoners e.g. having learning disabilities or problems with reading or writing. 


\section{Enter Figure 1 around here:}

\section{Characteristics of participants with ID}

The majority of participants who screened positive on the LDSQ were sentenced prisoners ( $n=16$; 89\%) with just two (11\%) being identified on remand before the prison rerolled from a category $\mathrm{B}$ to a category $\mathrm{C}$ prison.

Table 1: shows the characteristics of participants who screened positive on the LDSQ compared with those who screened negative for ND. There were statistically significant differences between those with and without ID on a range of socio-demographic measures.

Participants with ID were more likely to be of white ethnicity 14 (78\%), compared to 4 (22\%) from a black or minority ethnic (BME) background. The majority of the sample was young with 17(94\%) under 35 years old. . The ID group were more likely to have been single, homeless or unemployed and not have any qualifications before coming into prison and to have left school before the age of 16 .

\section{Enter Table 1 around here:}

\section{Co-occurring mental health problems and substance abuse}

There were mental health data for 16 of the 18 participants who screened positive on the LDSQ and 69 participants who screened negative for ND. Lifetime rates of mental health problems were significantly higher in prisoners who screened positive on the LDSQ compared with those who screened negative for ND (75\% ( $n=12)$ vs. $\left.43.5 \%(n=30) ; X^{2}=5.163, p=0.023\right)$.

Results from these groups are compared in Table 2 which shows that rates of current mental health problems were higher among those who screened positive on the LDSQ. This was significantly higher than participants without ND for anxiety, psychosis and antisocial personality disorder. Forty-four per cent of participants who screened positive on the LDSQ had a current mental health problem a further $37.5 \%$ had an alcohol or substance misuse problem.

\section{Enter Table 2 around here:}


Participants who screened positive on the LDSQ were significantly more likely to have a moderate to high level of suicidality $\left(37.5 \%(n=6)\right.$ vs. $\left.7 \%(n=5), X^{2}=10.552, p=0.001\right), 25 \%$ had thought about suicide in the last month and $63 \%$ had attempted suicide in the past. Furthermore, nearly 38\% had thought about self-harm in the last month and 19\% had injured themselves on purpose, see table 3.

\section{Enter Table 3 around here:}

\section{Offending and risk}

Prisoners who screened positive on the LDSQ were more likely to be housed on the vulnerable prisoners' (VP) wing (33\% vs. 17\%) but this was not statistically significant. There were two prisoners who screened positive on the LDSQ who were housed on the VP wing but had not been sentenced for a sexual offence indicating they were seen as vulnerable for another reason (usually because they were debtors and/or had been bullied on other prison wings). Prisoners who screened positive on the LDSQ were more likely to have been recorded by the prison as being at high risk of harm to themselves or from others $(56 \%(n=10))$ compared with those who screened negative for ND $(28 \%(n=43), p=0.017)$

Prisoners who screened positive on the LDSQ were more likely to have reported being in prison before compared with those who screened negative for ND $(90 \%(n=9)$ vs. $54 \%(n=77)$, $\mathrm{p}=0.026$ ). There were few significant differences in the offending characteristics of prisoners who screened positive on the LDSQ and those who screened negative for ND, as shown in Table 4.

\section{Enter Table 4 around here:}

\section{Co-occurring neurodevelopmental disorders}

Participants who screened positive on the LDSQ were more likely to screen positive for ADHD or ASD than those who screened negative ( $67 \%$ vs. $24 \%$, $p<0.001$ and $33 \%$ vs. $18 \%, p=0.112$ respectively). This result was only statistically significant for ADHD.

Eight participants (44\%) screened positive on the LDSQ and for one other ND and five (28\%) screened positive on the LDSQ, and for ADHD and ASD. Thus many prisoners who screened positive on the LDSQ appeared to have complex needs with significant problems with inattention, hyperactivity, impulsivity or social communication in addition to their cognitive impairments. 


\section{Discussion}

The rate of ID reported in this study (7.5\%) was similar to that found by previous research on sentenced prisoners (Talbot, 2008; Talbot, 2009). The LDSQ screening tool has a reported specificity and sensitivity of over $80 \%$ (McKenzie et al, 2012). In this study of the 18 who screened positive for ID, 16 self-reported a previous diagnosis. In clinical practice, in the absence of a screen, often clinicians will ask a direct question which may uncover need or diagnosis e.g., do you have an intellectual disability? Although this approach can identify some that may have been missed, it is worth bearing in mind that it can also provide a number of false positives and identify those without ID. In this study an additional 14 reported that they have ID or learning difficulties, who did not screen positive on the LDSQ. It is important to remember that prevalence figures are sensitive to methodological approaches employed and there is evidence to suggest that when a full diagnostic assessment is completed prevalence figures are reduced (Murphy et al, 2015). Fazel et al's, (2008) systematic review of 12,000 prisoners reported rates of between 0.5 and $1.5 \%$ of prisoners were diagnosed with ID.

This study found that the majority of prisoners with ID were white and their ages ranged from 18-35, whereas the control group was significantly older and were more representative of the local population. Although we would expect people with ID in prison to be mostly young men, it would be in line with the rest of the prison population. Although both those with ID and the controls had high rates of social disadvantage, the social demographic profiles of prisoners with ID found significantly higher rates of homelessness, unemployment and leaving school early without qualification, adding support to the theory that low intelligence combined with poor social economic status significantly increases risk of offending (Dickson, Emerson, \& Hatton, 2005). The higher rates of homelessness and poor educational attainment found was in line with current evidence relating to prisoners with ID (Ali \& Hassiotis, 2016).

Participants who screened positive for ID were more likely to screen positive for other NDs, with comorbid ADHD reaching significance. This group presented with additional problems including inattention, hyperactivity, impulsivity, poor social communication in addition to cognitive impairment associated with ID. The introduction of screening would allow this vulnerable group to be targeted, particularly as studies of ADHD in prisons have revealed a high correlation with offending with rates between $20-30 \%$ reported in a number of prison studies across countries (Young et al, 2015). So far studies into offending and ND in adults have tended to be disorder specific. This is not the case for children or young offenders where there is evidence of high rates of offending in those with ND and neurodisability (Hughes et al, 2012). However studies examining ND in adult prisoners are in their infancy (McCarthy et al, 2016), in spite of the evidence of significant comorbidity of these conditions and its relationship to offending in 
children (Hughes et $a$,; 2012). In addition to significant comorbidity between other NDs and ID, there are also high rates of comorbidity of ID and mental illness. However there is little evidence on the mental health of prisoners with ID. A systematic review of 33, 588 prisoners reported that $3.7 \%$ had a psychotic illness and 11.4\% major depression (Fazel \& Seewald 2012). This concurs with a number of studies that have found higher rates of mental illness in prisoners with ID (Dias et al, 2013 and Hassiotis et al, 2011). This study found rates of current mental illness at far higher rates following interview of prisoners by researchers using the MINI. There were significant differences for anxiety disorders, psychosis and anti-social personality disorder for ID prisoners compared to non ND controls as well as higher rates of depression and substance use disorders. The face to face interview employed in this study may be a way forward to provide greater accuracy of data, which could be limited by the use of other methods e.g., self-report scales as used in the Dias study or use of survey data (Hassiotis et al, 2011). These methods may underestimate the full extent of mental health problems given the issue of undetected mental health problems in people with ID and inherent difficulties that this population faces in describing their mental health difficulties and completing written questionnaires. The findings from this current study, when compared to Hassiotis and colleagues' paper (which used data from the Psychiatric Morbidity among Prisoners in England and Wales, 1997 survey) illustrates the lack of progress in addressing the mental health needs of prisoners with ID over the last 20 years.

Throughout the prison population, self-harm, suicidal thoughts and suicide attempts are an ongoing concern. Due to poor recognition of ID in prison the increased risk of suicide in this population is not appreciated, although research suggests they are at least as vulnerable and maybe at even greater risk of suicide ideation when compared to the general population (Salvatore et al, 2016). People with ID are more likely to have an increased suicide risk (Giannini et al, 2010). However it is difficult to get a consensus on rates of suicide mortality. Shaw, Appleby, \& Baker, (2003), reported that 3\% of prisoners who had committed suicide had ID. This is over twice the rate reported in a systematic review of 12000 prisoners (Fazel, et al, 2008). In the general population what evidence there is suggests decreased mortality for people with ID. A 35 year follow up study of 2369 Finnish people with ID, reported that the rate for completed suicides for people with ID was one-third of the general population rate Patja et al. (2001). The risk factors which increase the risk of suicide such as clinical depression, history of self-harm, unemployment, loneliness, unemployment, lack of support, early onset mental illness and being treatment resistive are the same as the general population (Mollison et al, 2014). The lack of screening for ID means this vulnerable group continue to go unrecognised and therefore are not represented in cases of completed suicide. The rates of self-harm and thoughts about self-harm, and thoughts about suicide and attempted suicide as well as number of life time 
suicide attempts were all significantly higher for ID prisoners compared to other prisoners without ND and at higher rates than the two-fold increase for ID prisoners compared to controls previously (Hassiotis et al, 2011).

Crime studies point to the fact that lower IQ is a predictor of crime, it is unclear from these studies of the prevalence of ID offending when social deprivation is considered (Murphy and Mason, 2014). The offence data for people with ID is sensitive to setting and level of security as there will be a greater prevalence of more serious offenders in higher security mental health services and prisons. For many years it has been put forward that people with ID were over represented in terms of certain offences such as sex offending and arson, despite evidence to the contrary (Hayes 1991, Gilbey et al, 1989).

There is evidence to suggest that in general the types of offences committed by people with ID are similar to other offenders (Murphy and Mason, 2014). In this study prisoners with ID did commit more sex offences, however this was not significant. The only significant difference found in the offence rates examined was for robbery, (ID prisoners $18 \% \mathrm{v}$ Non ND Prisoners $3 \%)$. In a study of remand prisoners Murphy et al. (1995) of 21 prisoners with ID and a control group of 21 prisoners, reported 9.5\% (ID) v 4.8\% (no ID) were accused of robbery. The high reported rates of robbery appear consistent with Hodgins' (1992) reporting a third of prisoners with ID had been convicted of theft. However, questions about the representativeness of the sample have affected the study's validity (Lindsay \& Dernevik, 2013) .

Studies of substance use and ID have shown contact with the Criminal Justice System (Taggart \& Chaplin, 2014; Chaplin et al, 2015, McGilvray et al, 2016). Given the increased awareness of substance use by people with ID, the lack of convictions for alcohol and substance abuse for prisoners with ID in this study is therefore surprising given the higher rates of substance use problems reported by ID prisoners, i.e., $32 \%$ compared to $26 \%$ of other prisoners during the MINI screen. This is not to say that crimes were not committed under the influence of alcohol. Research by Holland \& Persson (2011) also found lower rates of substance use offences in an Australian prison sample.

In general, there was a lack of awareness about ID among staff and prison healthcare services. There were no ID specialists working in the prison and no system of referral to local ID or social services on release. Improvements in prison health care should include better recognition of ID, and provision of appropriate assessment and treatment of co-occurring mental health symptoms. Currently, there is no regular programme of screening for ID although some staff were able to identify prisoners likely to have ID. However, by their own admission staff felt they 
lacked the knowledge and skills to offer appropriate support and interventions in the prison for this group. The research team facilitated a series of training sessions for staff. The feedback was positive and the staff were able to identify future training needs to help working with this group. Across prisons staff should receive training to improve their awareness and understanding of ID to inform everyday working with this group. In terms of mental health assessment, the mental health care staff should receive enhanced training on assessment and intervention for ID.

The Bradley Report (Ministry of Justice, 2009) recommended the need to put in place initiatives to improve the recognition of ID throughout the CJS, along with the provision of interventions that promote liaison and diversion. The findings of the study confirm the continuing failure to identify people with ID early in the CJS. Further work is needed to ensure that national guidance and policy is being effectively implemented. This should be achieved through the development of a new integrated pathway through the CJS for people with ID by establishing partnerships between health/social care providers and offender management services. There is currently a lack of release planning and signposting to access support outside. Access to formal support from health and social care services is also difficult to achieve for a number of reasons including poor recognition of people with ID, a lack of partnership working and interfaces with the CJS and not being eligible to receive services.

Eligibility criteria for services for people with ID are often an issue that impacts on CJS, local mental health and social services working. As a consequence, prisoners with ID are likely to remain in custody for far longer than is necessary. Due to difficulty in accessing local health and social service provisions many offenders with ID are still placed out of area. For many, it is the 'forensic' label that appears to prevent access to local services. This continues to occur despite evidence that the needs of those who end up placed out of area are no different to those who remain 'in area' (Chaplin et al, 2010). This needs to be addressed given the human and financial costs of exporting people far from home. The findings from this study illustrate that ID prisoners are at increased risk of mental and physical health problems, are at a higher risk of suicide and who are more likely to be convicted and receive a custodial sentence than other prisoners without ND. The problem of not identifying people with ID once in prison is a huge one and can affect all aspects of prison life and for some mean that chances of release or parole are lessened as they will not have the cognitive ability to engage in prison programmes which may have a bearing on parole and potential release. In moving this agenda forward, a realistic approach is called for. Currently in prison it would be difficult to offer a full diagnostic assessment for people thought to have ID likewise these services are noften not commissioned for adults outside of prison. However routine screening should be a goal we can aspire to within prisons. Although there are educated estimates of the likely number of prisoners, there is a lack of criminological 
research which examines pathways and sentencing patterns within the Criminal Justice System. Although there is increasing research globally in this area it may be difficult to generalise outcomes across different legal systems.

More needs to be done at a young age given the evidence of ND in young offender populations. This is more important given that nearly half of those with ID screened positive for another ND. This and the poor social economic status leaves this group vulnerable unless more support and early intervention is offered.

\section{Limitations}

As mentioned previously the robustness of finding can be compromised by different methodologies. One issue which is particularly relevant to prison studies is the issue of recruitment and retention. Whilst it is always preferable to have a uniform database in terms of participant numbers,prison life can make this all but impossible (due to prisoners being released or transferred to other establishments) adding to the attrition rate that might be expected in a clinical study. In the absence of total prison population studies, controlled comparison of ID with other groups of prisoners is a legitimate method to employ. Although it is important to say this study allows comparison of the different groups ID v Non ND, and is not a prevalence study as participants were mainly selected on the basis of which prisoners could be easily located, as such, it is possible that the sample we recruited were less engaged with education or work, less likely to be associating with other prisoners and were therefore more likely to be in their cell. Although the screening for ID using the LDSQ was able to identify prisoners with ID but like as Murphy et al (2015) noted it is not designed to offer assessment of abilities that a full diagnostic assessment would provide. Given the studies small sample size of prisoners with ID and use of a single setting affects the generalisability of what can be inferred from the results and more research needs to be completed using larger numbers to get a greater understanding.

\section{Conclusion}

A considerable number of prisoners had impairments in cognitive and social functioning and those with ID were found to have high levels of mental illness. There is little research about the characteristics and experiences of prisoners with ID. This study highlights a number of areas where services could be improved through skills and awareness training to manage and support individuals with ID throughout the CJS. The project highlights major gaps in current healthcare provision both in prison and within local mental health services, which has an enormous human cost and significant financial burden which impacts on the provision of local health and social services. 
In terms of current health and social care arrangements within the prison system, the lack of screening and poor identification of people with ID can have a number of potential consequences for those not identified, e.g., referral to treatment programmes where the person does not have the cognitive ability required to participate. There is also a lack of provision to support prisoners pre-release. Many will be unable to access practical support on release, which is likely to increase the risk of reoffending. The research team heard numerous stories suggesting this was the case. We found the majority (80\%) had been in prison before. This was not out of intent or defiance, but through a lack of understanding of what was expected of them, either because the language was too complex, they lacked the skills to find out where they were going or were unable to read or understand written instructions. Without appropriate support this group will continue to find themselves trapped within the system.

The study demonstrates that early recognition on first contact with the CJS can potentially have significant consequences on how we treat and view people with ID who offend or are accused of offending. One solution may be to direct more resources at the Court stage to identify people with ID. This would allow experts to make recommendations of informed appropriate noncustodial sentencing options. However, Court Liaison \& Diversion aimed to identify people with ID is in its infancy and only an aspiration in many areas. What systems there are, are currently focussed towards the diversion of those with mental illness. These services need to be extended to provide expertise to identify ID and other NDs. What is clear is the need for staff working across the CJS to be able to access training on ID. Currently, many feel ill equipped and feel they lack the necessary skills to work with this group. As part of this project a number of study sessions were held for staff and prisoners at the prison which were well-received, however this did not stop demand for support with this group as seen by the number of referrals or opinions requested by prison healthcare. This study has concentrated on identifying the characteristics of prisoners with ID and their increased vulnerability and illustrates how poorly we support the most vulnerable in society. Prisoners in general are a vulnerable group and those with ID even more so. For prisoners with ID there needs to be more support both whilst in prison and postrelease. Currently prisoners are not signposted to services on release or given information on how to access support. It is not known how many, but in the authors experience too many people with ID are recalled to prison for unintentionally breaking conditions of their release which they are either unable to understand or are unrealistic for them to meet without support. 


\section{References}

Adler, L., Kessler, R. C., \& Spencer, T. (2003). Adult ADHD Self-Report Scale (ASRS-v1.1) Symptom Checklist.

Ali, A., Ghosh, S., Strydom, A. \& Hassiotis, A., 2016. Prisoners with intellectual disabilities and detention status. Findings from a UK cross sectional study of prisons. Research in developmental disabilities, 53, pp.189-197.

Brugha, T. S., McManus, S., Smith, J., Scott, F. J., Meltzer, H., Purdon, S., . . Bankart, J. (2012). Validating two survey methods for identifying cases of autism spectrum disorder among adults in the community. Psychol Med, 42(3), 647-656.

Chaplin, E., Kelisdi, K., Emery, H., O’Hara, J., Lockett, J., \& McCarthy, J. (2010). People with learning disabilities placed out of area - the south London experience. Offending and Learning Disabilities Journal, 1, 3-14.

Chaplin, E., McCarthy, J \& Underwood, L (2013) Autism spectrum conditions and offending: An introduction to the special edition, Journal of Intellectual Disabilities and Offending Behaviour, $4: 1 / 2,5-8$

Chaplin, E., Partsenidis, I., Samuriwo, B., Underwood, L. \& McCarthy, J. (2015). Does substance use predict contact with the criminal justice system for people with intellectual disabilities? Journal of intellectual disabilites and offending behaviour 5, 147-153.

Dias, S., Ware, R.S., Kinner, S.A. \& Lennox, N.G., 2013. Co-occurring mental disorder and intellectual disability in a large sample of Australian prisoners. Australian and New Zealand journal of psychiatry, 47(10), pp.938-944.

Dickson, K., Emerson, E., \& Hatton, C. (2005). Selfreported anti-social behaviour: Prevalence and risk factors amongst adolescents with and without intellectual disability. Journal of Intellectual Disability Research, 49, 820-826. 
Fazel, S. \& Seewald, K., 2012. Severe mental illness in 33588 prisoners worldwide: systematic review and meta-regression analysis. The British Journal of Psychiatry, 200(5), pp.364-373.

Fazel, S., Xenitidis, K \& Powell, J. (2008). The prevalence of intellectual disabilities among 12000 prisoners: a systematic review. International Journal of Law \& Psychiatry, 31, 369- 373.

Giannini, M., Bergmark, B., Kreshover, S., Elias, E., Plummer, C. \& O’Keefe, E. (2010). Understanding suicide and disability through three major disabling conditions: Intellectual disability, spinal cord injury, and multiple sclerosis. Disability \& Health Journal, 3(2), 74-78

Gilby, R., Wolf, L. \& Goldberg, B., 1989. Mentally retarded adolescent sex offenders. A survey and pilot study. Canadian journal of psychiatry. Revue canadienne de psychiatrie, 34(6), pp.542-548.

Harris, J.C. (2006). Intellectual disability. Understanding its development, causes, classification, evaluation and treatment. Oxford: Oxford University Press.

Hassiotis, A., Gazizova, D., Akinlonu, L., Bebbington, P., Meltzer, H., \& Strydom, A. (2011). Psychiatric morbidity in prisoners with intellectual disabilities: analysis of prison survey data for England and Wales. British Journal of Psychiatry, 199(2), 156-157.

Hayes, S., 1991. Sex offenders. Australia and New Zealand Journal of Developmental Disabilities, 17(2), pp.221-227.

Her Majesty's Chief Inspector of Prisons. (2013). Report on an unannounced full follow-up inspection of HMP Brixton 1-12 July 2013 by HM Chief Inspector of Prisons. London.

Hodgins S. (1992) Mental disorder, intellectual deficiency and crime: evidence from a birth cohort. Archives of General Psychiatry 49, 476-483.

Holland, S. \& Persson, P., (2011). Intellectual disability in the Victorian prison system: Characteristics of prisoners with an intellectual disability released from prison in 2003-2006. Psychology, Crime \& Law, 17(1), pp.25-41. 
Hughes, N., Williams H, Chitsabesan, P, Davies R, Mounce L. (2012) Nobody Made the Connection: The Prevalence of Neurodisability in Young People Who Offend. London, United Kingdom: Office of the Children's Commissioner for England.

Lindsay, W. R. \& Dernevik, M. (2013), Risk and offenders with intellectual disabilities: reappraising Hodgins (1992) classic study. Crim Behav Ment Health, 23: 151-157. doi:10.1002/cbm.1862

McCarthy, J., Chaplin, E., Underwood, L., Forrester, A., Hayward, H., Sabet, J., Young, S., Asherson, P., Mills, R. \& Murphy, D., 2016. Characteristics of prisoners with neurodevelopmental disorders and difficulties. Journal of Intellectual Disability Research, 60(3), pp.201-206.

McGillivray, J. A., \& Moore, M. R. (2001). Substance use by offenders with mild intellectual disability. Journal of Intellectual \& Developmental Disability, 26(4), 297-310. doi:10.1080/13668250120087317

McKenzie, K., Michie, A., Murray, A., \& Hales, C. (2012). Screening for offenders with an intellectual disability: The validity of the Learning Disability Screening Questionnaire. Research in Developmental Disabilities, 33(3), 791-795.

Ministry of Justice (2009) Lord Bradley's report on people with mental health problems or learning disabilities in the criminal justice system: the government's response. Ministry of Justice, London.

Ministry of Justice (2014). Offender Management Statistics Bulletin, Quarterly - October to December 2013

(https://www.gov.uk/government/uploads/system/uploads/attachment data/file/305741/off ender-management-statistics-october-december-2013.pdf).

Mollison., E Chaplin., E Underwood., L \& McCarthy, J (2014) A review of risk factors associated with suicide in adults with intellectual disability", Advances in Mental Health and Intellectual 
Disabilities, 8 (5),.302 - 308

Murphy, G. H., Gardner, J. \& Freeman, M. J. (2015), Screening Prisoners for Intellectual

Disabilities in Three English Prisons. J Appl Res Intellect Disabil. doi:10.1111/jar.12224

Murphy G. H., Harnett H. \& Holland A. J. (1995) Asurvey of intellectual disabilities amongst men onremand in prison. Mental Handicap Research 8, 81-98.

Murphy, G., \& Mason, J. (2014). People with intellectual disabilities and offending behaviours. In E. Tsakanikos \& J. McCarthy (Eds.), Handbook of psychopathology in adults with developmental and intellectual disabilities: Research, policy and practice . New York, NY: Springer Science.

NHS England (2015) Building the Right Support, A national plan to develop community services and close inpatient facilities for people with a learning disability and/or autism who display behaviour that challenges, including those with a mental health condition. NHS England: London

Patja, K., Mölsä, P. \& Iivanainen, M., (2001). Cause-specific mortality of people with intellectual disability in a population-based, 35-year follow-up study. Journal of Intellectual Disability Research, 45(1), pp.30-40.

Salvatore, T., Brow, J., Huntley, D., Kivisalu, T., Arndt, C., Wiley, C. Intellectual Disability and Suicide Risk: An Exploratory Discussion. The Journal of Special Populations, North America, 1, jun. 2016. Available at: <http://www.jghcs.info/index.php/jsp/article/view/493>. Date accessed: 11 Oct. 2016.

Shaw, J., Appleby, L. \& Baker, D. (2003) Safer Prisons - A National Study of Prison Suicides 19992000 by the National Confidential Inquiry into Suicides and Homicides by People with Mental Illness. London: Department of Health.

Sheehan, D., Lecrubier, Y., Janavs, J., Sheehan, K., Gray, C., Weiller, E., . . Even, C. (2010). M.I.N.I. Mini International Neuropsychiatric Interview. English version 6.0.0 DSM-IV. 
Singleton, N., Meltzer, H., Gatward, R., Coid, J., \& Deasy, D. (1998). Psychiatric morbidity among prisoners: Summary report. London, The Stationery Office.

Straw, A. \& Lomri, S. (2010). Public law update: failing prisoners with learning disabilities. Criminal Law and Justice Weekly, 174, 292-3.

Taggart, L. \& Chaplin, E. (2014) Substance abuse, Ch. 14, pp 205-226 In Handbook of Psychopathology in Intellectual Disability (Tsakanikos, E. \& McCarthy, J. Eds.) Springer, New York

Talbot, J. (2007). Identifying and supporting prisoners with learning difficulties and learning disabilities: the views of prison staff. London: Prison Reform Trust.

Talbot, J. (2008). No one knows report and final recommendations: Prisoners voices Experiences of the criminal justice system by prisoners with learning disabilities and difficulties. London: Prison Reform Trust.

Young, S., Moss, D., Sedgwick, O., Fridman, M., \& Hodgkins, P. (2015) A meta-analysis of the prevalence of attention deficit hyperactivity disorder in incarcerated populations. Psychological Medicine, 45(02), 247-258 http://dx.doi.org/10.1017/S0033291714000762 